\title{
An exact approach for the consistent vehicle routing problem (ConVRP)
}

\author{
Barros, L. ${ }^{a}$, Linfati, R. ${ }^{b}$, Escobar, J.W., ${ }^{\text {c, }}$ \\ ${ }^{a}$ Escuela de Ingeniería Industrial, Universidad del Bío-Bío, Chile, Concepción, Chile \\ ${ }^{\mathrm{b}}$ Departamento de Ingeniería Industrial, Universidad del Bío-Bío, Chile, Concepción, Chile \\ 'Department of Accounting and Finance, Faculty of Business Management, Universidad del Valle, Cali, Colombia
}

\begin{abstract}
A B S T R A C T
This paper proposes a mathematical model for the Consistent Vehicle Routing Problem (ConVRP). The ConVRP is an extension of the VRP, considering customer satisfaction through consistent service. The consistency may be based on time or on the vehicle that offers the service. This paper proposes a novel mathematical model that allows solving the ConVRP for several companies for which visits to the customers need to be from the same service provider (namely, the same vehicle and driver). The efficiency of the model is tested on structured instances by changing customer distribution (uniform or clustered), depot location, and arrival time to the customer and removing certain constraints to see if they affect the performance of the objective function. The mathematical model is flexible and could be adapted to any characteristic of instances. The model was developed in the AMPL programming language and solved with the solvers CPLEX and Gurobi. The results are promising based on the efficiency of the proposed method at solving the problem.
\end{abstract}

(C) $2020 \mathrm{CPE}$, University of Maribor. All rights reserved.

\author{
ARTICLE INFO \\ Keywords: \\ Vehicle routing problem (VRP); \\ Consistent vehicle routing (ConVRP); \\ Mathematical model; \\ Mixed integer linear programming model; \\ Optimization; \\ Exact algorithms; \\ Modelling; \\ CPLEX; \\ Gurobi \\ *Corresponding author: \\ john.wilmer.escobar@correounivalle.edu.co \\ (Escobar, J.W.)
}

Article history:

Received 24 March 2020

Revised 10 October 2020

Accepted 13 October 2020

\section{References}

[1] Wu, Q., Wang, X., He, Y.D., Xuan, J., He, W.D. (2018). A robust hybrid heuristic algorithm to solve multi-plant milkrun pickup problem with uncertain demand in automobile parts industry, Advances in Production Engineering \& Management, Vol. 13, No. 2, 169-178, doi: 10.14743/apem2018.2.282.

[2] Giaglis, G.M., Minis, I., Tatarakis, A., Zeimpekis, V. (2004). Minimizing logistics risk through real-time vehicle routing and mobile technologies: Research to date and future trends, International Journal of Physical Distribution \& Logistics Management, Vol. 34, No. 9, 749-764, doi: 10.1108/09600030410567504.

[3] Feillet, D., Garaix, T., Lehuédé, F., Péton, O., Quadri, D. (2014). A new consistent vehicle routing problem for the transportation of people with disabilities, Networks, Vol. 63, No. 3, 211-224, doi: 10.1002/net.21538.

[4] Somboonwiwat, T., Khompatraporn, C., Miengarrom, T., Lerdluechachai, K. (2018). A bi-objective environmentaleconomic optimisation of hot-rolled steel coils supply chain: A case study in Thailand, Advances in Production Engineering \& Management, Vol. 13, No. 1, 93-106, doi: 10.14743/apem2018.1.276.

[5] Tarantilis, C.D., Stavropoulou, F., Repoussis, P.P. (2012). A template-based tabu search algorithm for the consistent vehicle routing problem, Expert Systems with Applications, Vol. 39, No. 4, 4233-4239, doi: 10.1016/j.eswa. 2011.09.111.

[6] Bernal, J., Escobar, J.W., Paz, J.C., Linfati, R., Gatica, G. (2018). A probabilistic granular tabu search for the distance constrained capacitated vehicle routing problem, International Journal of Industrial and Systems Engineering, Vol. 29, No. 4, 453-477, doi: 10.1504/IJISE.2018.094267. 
[7] Escobar, J.W. (2014). Heuristic algorithms for the capacitated location-routing problem and the multi-depot vehicle routing problem, 4OR - A Quarterly Journal of Operations Research, Vol. 12, 99-100, doi: 10.1007/ s10288-013-0241-4.

[8] Escobar, J.W., Linfati, R., Toth, P., Baldoquin, M.G. (2014). A hybrid granular tabu search algorithm for the multidepot vehicle routing problem, Journal of Heuristics, Vol. 20, No. 5, 483-509, doi: 10.1007/s10732-014-9247-0.

[9] Schulze, J., Fahle, T. (1999). A parallel algorithm for the vehicle routing problem with time window constraints, Annals of Operations Research, Vol. 86, 585-607, doi: 10.1023/A:1018948011707.

[10] Jozefowiez, N., Semet, F., Talbi, E.-G. (2008). Multi-objective vehicle routing problems, European Journal of Operational Research, Vol. 189, No. 2, 293-309, doi: 10.1016/j.ejor.2007.05.055.

[11] Bolaños, R.I., Escobar, J.W., Echeverri, M.G. (2018). A metaheuristic algorithm for the multi-depot vehicle routing problem with heterogeneous fleet, International Journal of Industrial Engineering Computations, Vol. 9, No. 4, 461-478, doi: 10.5267/j.ijiec.2017.11.005.

[12] Gaudioso, M., Paletta, G. (1992). A heuristic for the periodic vehicle routing problem, Transportation Science, Vol. 26, No. 2, 86-92, doi: $10.1287 / \operatorname{trsc} .26 .2 .86$.

[13] Min, J.N., Jin, C., Lu, L.J. (2019). Maximum-minimum distance clustering method for split-delivery vehicle-routing problem: Case studies and performance comparisons, Advances in Production Engineering \& Management, Vol. 14, No. 1, 125-135, doi: 10.14743/apem2019.1.316.

[14] Sepúlveda, J., Escobar, J.W., Adarme-Jaimes, W. (2014). An algorithm for the routing problem with split deliveries and time windows (SDVRPTW) applied on retail SME distribution activities, DYNA, Vol. 81, No. 187, 223-231, doi: 10.15446/dyna.v81n187.46104.

[15] Bernal, J., Escobar, J.W., Linfati, R. (2017). A granular tabu search algorithm for a real case study of a vehicle routing problem with a heterogeneous fleet and time windows, Journal of Industrial Engineering and Management, Vol. 10, No. 4, 646-662, doi: 10.3926/jiem.2159.

[16] Stewart Jr., W.R., Golden, B.L. (1983). Stochastic vehicle routing: A comprehensive approach, European Journal of Operational Research, Vol. 14, No. 4, 371-385, doi: 10.1016/0377-2217(83)90237-0.

[17] Dumas, Y., Desrosiers, J., Soumis, F. (1991). The pickup and delivery problem with time windows, European Journal of Operational Research, Vol. 54, No. 1, 7-22, doi: 10.1016/0377-2217(91)90319-Q.

[18] Santa Chávez, J.J., Escobar, J.W., Echeverri, M.G., Meneses, C.A.P. (2018). A heuristic algorithm based on tabu search for vehicle routing problems with backhauls, Decision Science Letters, Vol. 7, No. 2, 171-180, doi: 10.5267/ j.dsl.2017.6.001.

[19] Cao, Q.K., Yang, K.W., Ren, X.Y. (2017). Vehicle routing optimization with multiple fuzzy time windows based on improved wolf pack algorithm, Advances in Production Engineering \& Management, Vol. 12, No. 4, 401-411, doi: 10.14743/apem2017.4.267.

[20] Linfati, R., Escobar, J.W. (2018). Reoptimization heuristic for the capacitated vehicle routing problem, Journal of Advanced Transportation, Vol. 2018, Article ID 3743710, doi: 10.1155/2018/3743710.

[21] Groër, C., Golden, B., Wasil, E. (2009). The consistent vehicle routing problem, Manufacturing \& Service Operations Management, Vol. 11, No. 4, 630-643, doi: 10.1287/msom.1080.0243.

[22] Linfati, R., Escobar, J.W., Escalona, J. (2018). A two-phase heuristic algorithm for the problem of scheduling and vehicle routing for delivery of medication to patients, Mathematical Problems in Engineering, Vol. 2018, Article ID 8901873, doi: 10.1155/2018/8901873.

[23] Den Ridder, R. (2014). ConRTR, a consistent vehicle routing problem algorithm, from https://pdfs.semanticscholar.org/3a72/80ff17eaf4938b1fe7f4944e5c352a90d0e6.pdf accessed September 12, 2019.

[24] Lehuédé, F., Pavageau, C., Péton, O. (2008). Un système d'aide à la décision pour planifier les transports vers les établissements médico-sociaux, In: Proceedings of 5è conférence Handicap, Paris, France, 168-173.

[25] Lespay, H., Suchan, K. (2019). The consistent vehicle routing problem for a food distribution firm, In: Proceedings of Verolog Conference, Workshop of the EURO Working Group on Vehicle Routing and Logistics Optimization, Seville, Spain, 1-2.

[26] Campelo, P., Neves-Moreira, F., Amorim, P., Almada-Lobo, B. (2019). Consistent vehicle routing problem with service level agreements: A case study in the pharmaceutical distribution sector, European Journal of Operational Research, Vol. 273, No. 1,131-145, doi: 10.1016/i.ejor.2018.07.030.

[27] Macdonald, T., Dörner, K., Gandibleux, X. (2009). Metaheuristics for the consistent nurse scheduling and routing problem, from http://oro.univ-nantes.fr/sujets-09-10/macdonald.pdf accessed September 20, 2019.

[28] Goeke, D., Roberti, R., Schneider, M. (2019). Exact and heuristic solution of the consistent vehicle-routing problem, Transportation Science, Vol. 53, No. 4, 986-1000, doi: 10.1287/trsc.2018.0864.

[29] Xu, Z., Cai, Y. (2018). Variable neighborhood search for consistent vehicle routing problem, Expert Systems with Applications, Vol. 113, 66-76, doi: 10.1016/j.eswa.2018.07.007.

[30] Stavropoulou, F., Repoussis, P.P., Tarantilis, C.D. (2019). The vehicle routing problem with profits and consistency constraints, European Journal of Operational Research, Vol. 274, No. 1, 340-356, doi: 10.1016/j.ejor.2018. $\underline{09.046}$.

[31] Kovacs, A.A., Golden, B.L., Hartl, R.F., Parragh, S.N. (2015). The generalized consistent vehicle routing problem, Transportation Science, Vol. 49, No. 4, 796-816, doi: 10.1287/trsc.2014.0529.

[32] Kovacs, A.A., Parragh, S.N., Hartl, R.F. (2015). The multi-objective generalized consistent vehicle routing problem, European Journal of Operational Research, Vol. 247, No. 2, 441-458, doi: 10.1016/j.ejor.2015.06.030.

[33] Miller, C.E., Tucker, A.W., Zemlin, R.A. (1960). Integer programming formulation of traveling salesman problems, Journal of the ACM, Vol. 7, No. 4, 326-329, doi: 10.1145/321043.321046. 
[34] Uchoa, E., Pecin, D., Pessoa, A., Poggi, M., Vidal, T., Subramanian, A. (2017). New benchmark instances for the capacitated vehicle routing problem, European Journal of Operational Research, Vol. 257, No. 3, 845-858, doi: 10.1016/j.ejor.2016.08.012.

[35] Zhao, P.X., Luo, W.H., Han, X. (2019). Time-dependent and bi-objective vehicle routing problem with time windows, Advances in Production Engineering \& Management, Vol. 14, No. 2, 201-212, doi: 10.14743/apem2019. $\underline{2.322 .}$.

[36] Arab, R., Ghaderi, S.F., Tavakkoli-Moghaddam, R. (2020). Two efficient meta-heuristic algorithms for the robust inventory routing problem with backhaul, Tehnički Vjesnik - Technical Gazette, Vol. 27, No. 3, 793-802, doi: 10.17559/TV-20180814091028.

[37] Gocken, T., Yaktubay, M. (2019). Comparison of different clustering algorithms via genetic algorithm for VRPTW, International Journal of Simulation Modelling, Vol. 18, No. 4, 574-585, doi: 10.2507/IJSIMM18(4)485.

[38] Sepúlveda, J., Escobar, J.W., Adarme-Jaimes, W. (2014). An algorithm for the routing problem with split deliveries and time windows (SDVRPTW) applied on retail SME distribution activities, DYNA, Vol. 81, No. 187, 223-231, doi: 10.15446/dyna.v81n187.46104.

[39] Santa Chávez, J.J., Escobar, J.W., Echeverri, M.G., Meneses, C.A.P. (2018). A heuristic algorithm based on tabu search for vehicle routing problems with backhauls, Decision Science Letters, Vol. 7, No. 2, 171-180, doi: 10.5267/ j.dsl.2017.6.001.

[40] Santa Chávez, J.J., Echeverri, M.G., Escobar, J.W., Meneses, C.A.P. (2015). A metaheuristic ACO to solve the multidepot vehicle routing problem with backhauls, International Journal of Industrial Engineering and Management (IJIEM), Vol. 6, No. 2, 49-58. 


\title{
APEM
}

\section{Natančen pristop za problem doslednega usmerjanja vozil (ConVRP)}

\author{
Barros, L. ${ }^{a}$, Linfati, R. ${ }^{b}$, Escobar, J.W., ${ }^{c,}$ \\ aEscuela de Ingeniería Industrial, Universidad del Bío-Bío, Chile, Concepción, Chile \\ bDepartamento de Ingeniería Industrial, Universidad del Bío-Bío, Chile, Concepción, Chile \\ 'Department of Accounting and Finance, Faculty of Business Management, Universidad del Valle, Cali, Colombia
}

\begin{abstract}
POVZETEK
$\mathrm{V}$ prispevku je predlagan matematični model za problem konsistentnega usmerjanja vozil (ConVRP). ConVRP je razširitev VRP, ki upošteva zadovoljstvo strank z doslednimi storitvami. Doslednost lahko temelji na času ali na vozilu, ki ponuja storitev. V prispevku je predlagan nov matematični model, ki omogoča reševanje ConVRP za več podjetij, pri katerih morajo biti obiski strank opravljeni s strani istega ponudnika storitev (istega vozila in voznika). Učinkovitost modela je bila preizkušena na strukturiranih primerih s spreminjanjem porazdelitve kupcev (enakomerne ali gručaste), lokacije skladišča in časa prihoda do stranke ter z odstranjevanjem nekaterih omejitev, da se ugotovi, ali le-te vplivajo na rezultat ciljne funkcije. Matematični model je prilagodljiv in ga je mogoče prikrojiti na kateri koli karakteristični primer. Model je bil razvit v programskem jeziku AMPL in rešen z reševalnikoma CPLEX in Gurobi. Glede na učinkovitost predlagane metode pri reševanju težave so rezultati obetavni.
\end{abstract}

(c) 2020 CPE, University of Maribor. All rights reserved.
PODATKI O ČLANKU

Ključne besede:

Problem usmerjanja vozil (VRP);

Konsistentno usmerjanje vozil (ConVRP);

Matematični model;

Mešani celoštevilski model linearnega programiranja;

Optimizacija;

Natančni algoritmi;

Modeliranje;

CPLEX;

Gurobi

*Kontaktna oseba:

john.wilmer.escobar@correounivalle.edu.co (Escobar, John Willmer)

Zgodovina članka:

Prejet 24. marca 2020

Popravljen 10. oktobra 2020

Sprejet 13. oktobra 2020 